\title{
EL PROCESO DE COMPRENSIÓN EN UNA LENGUA EXTRANJERA: UNA PROPUESTA PARA EVALUAR ESTRATEGIAS DE LECTURA
}

\section{(THE READING COMPREHENSION PROCESS IN A FOREIGN LANGUAGE: A PROPOSAL TO EVALUATE READING STRATEGIES)}

Eugenio Hidalgo Díez

Universidad de Granada, España

Mirtha Manzano Díaz

Universidad de Ciego de Ávila, Cuba

DOI: 10.5944/educxx1.17.1.10716

\section{Cómo referenciar este artículo/How to reference this article:}

Hidalgo Díez, E\y Manzano Díaz, M. (2014). El proceso de comprensión en una lengua extranjera: Una propuesta para evaluar estrategias de lectura. Educación XX1, 17 (1), 미-326. doi: 10.5944/educxx1.17.1.10716.

Hidalgo Díez, E $\square$ y Manzano Díaz, M. (2014). The reading comprehension process in a foreign language: a proposal to evaluate reading strategies. Educación XX1, 17 (1), प]9-326. doi: 10.5944/educxx1.17.1.10716.

\section{RESUMEN}

Consideramos que las estrategias para el aprendizaje de la lectura, no son directamente observables pero si detectables y medibles. La evaluación es un proceso que está siendo estudiado desde nuevas perspectivas mucho más conscientes, centradas en el individuo que aprende a aprender. Para evaluar el uso de estrategias durante la comprensión lectora hemos elaborado un cuestionario que analiza los procesos cognitivos y afectivos en relación con este proceso en una lengua extranjera. El procesamiento de la información escrita incluye una lista de destrezas que involucran no solo la decodificación del texto, sino también la aplicación y extensión de la información a otros contextos, y con ello la construcción de nuevos significados y sentidos - la creación de nuevos textos. Por tal motivo en su construcción, hemos tenido en cuenta las siguientes dimensiones: Conocimiento previo, nivel de traducción, nivel de interpretación y nivel de extrapolación. Se establecieron las características técnicas del instrumento por medio de los estadísticos Alpha de Crombach para la fiabilidad y juicio de expertos y análisis factorial para la validez, que vinieron a corroborar la bondad del cuestionario, lo que nos permite evaluar y autoevaluar el uso de estrategias de lectura en el proceso de enseñanzaaprendizaje en una lengua extranjera. 


\section{ABSTRACT}

We understand that learning strategies in the reading comprehension process are not directly observed, but they may be detectable and measurable. The evaluation is a process that is being studied from new angles more consciously focused on the person who learns how to learn. To assess the use of strategies during reading we have elaborated an instrument - a questionnaire aimed at evaluating the students' own cognitive and affective procedures through the reading comprehension process in a foreign language. The process of the information includes a list of skills which contains not only decoding, but also applying and extending the interpretation of the written text to other contexts, and the construction of new meaning and senses i. e. the creation of new texts. Therefore, after an exhaustive bibliographical review, four categories with their corresponding items were established: previous knowledge, translation level, interpretation level, and extrapolation level. Finally, we set up the technical characteristics of the questionnaire through statistic methods such as: Alpha of Crombach to validate its reliability, the criterion of experts and factorial analysis which confirmed its validity to evaluate the use of reading strategies in the teaching learning process of a foreign language.

\section{INTRODUCCIÓN}

Aunque los profesores de lengua, generalmente, hablamos en términos de habilidades lingüísticas o comunicativas (expresión oral y escrita y comprensión auditiva y de lectura), la realidad es que no siempre somos conscientes de que a través de ellas se desarrollan otras habilidades intelectuales y científicas y de que se establecen valores necesarios para el graduado de cualquier campo del saber, por eso, actualmente, se incluyen en los planes de estudio como competencias que se precisan para la formación de un profesional. Estas competencias determinan el verdadero propósito de la comunicación y la actividad cognoscitiva (Hidalgo, 2007). Nuestra investigación está dirigida a la comprensión de la lectura, pero sustentada sobre la base de que forma parte de la lengua como una entidad, pues el lenguaje, aunque metodológicamente lo separemos para su estudio, no es solo palabra unida, el lenguaje es sentido e intención y tiene, además de su carácter universal, su propia identidad,por lo tanto, también es contexto.

Nuestro estudio asume una posición compleja y sistémica del proceso de enseñanza aprendizaje de una lengua extranjera, por lo que entendemos que las limitaciones que confrontamos en la propia concepción de la enseñanza-aprendizaje de la lengua como un todo, son aplicables, también, al desarrollo de la competencia de lectura como parte esencial de la competencia comunicativa. En este sentido, consideramos que la lectura, además 
de objetivo y contenido, es medio, método y evaluación en el proceso de enseñanza-aprendizaje.

Igualmente, consideramos que la concepción de la lectura como instrumento didáctico de los aspectos lingüísticos, y no como herramienta que desarrolla la formación en la sistematización de los contenidos y en la adquisición de una cultura integral, es la que ha frenado la formación adecuada en la competencia comunicativa a nuestros futuros profesionales, dando lugar a que solo aquellos con una elevada motivación e independencia cognoscitiva alcancen los mejores resultados en la formación académica en una lengua extranjera (Manzano, 2009).

El artículo presenta el proceso seguido en la elaboración y validación de un cuestionario de evaluación-autoevaluación de estrategias de lectura. Se parte desde una posición epistemológica que revela la significación que adquiere la evaluación en este proceso, y se concluye con la dinámica que promueve este instrumento como medio y método de enseñanza-aprendizaje, durante el proceso de comprensión en una lengua extranjera.

\section{LA EVALUACIÓN DE LAS ESTRATEGIAS COMO COMPONENTE ESENCIAL DE LA ACTIVIDAD COGNOSCITIVA}

Cuando hablamos del proceso de enseñanza-aprendizaje de una lengua extranjera nos estamos refiriendo a la actividad cognoscitiva. Cuando nos referimos a las habilidades de la actividad verbal (lingüísticas), estamos haciendo referencia a la expresión y comprensión oral y escrita, a las que también denominamos destrezas comunicativas. Si vamos a la esencia de esas acciones (destrezas o habilidades), no podemos obviar los procesos mentales que durante las mismas ocurren, ni obviar operaciones o estrategias que el ser humano necesita para lograr el objetivo de determinada tarea.

Para desarrollar una actividad cognoscitiva, se precisa desplegar todo un sistema de acciones y operaciones dirigidas a un objetivo, en condiciones tanto internas como externas, en las que intervienen las esferas motivacional-afectiva y cognitivo-instrumental.

Las estrategias forman parte de la actividad cognoscitiva como método, ya que entran dentro del sistema de acciones de las habilidades a desarrollar en el proceso de enseñanza-aprendizaje. Igualmente, las estrategias son las operaciones que se realizan para lograr la acción o tarea a realizar durante la actividad. 
Es la propia vorágine del desarrollo científico-técnico en la actualidad, la que ha llevado a las ciencias de la educación a que tomemos conciencia de la necesidad del dominio de herramientas que permitan al profesional gestionar el conocimiento de forma autónoma, así como a desarrollar estudios que den respuesta a esta problemática.

En este sentido, la nueva universidad no puede transmitir todo el conocimiento desarrollado por la humanidad, por lo que se necesitan estrategias para poder interactuar con el conocimiento de manera independiente; por ello, los nuevos modelos profesionales exigen el manejo y dominio consciente de determinadas competencias que consoliden la integración de conocimientos, habilidades, actitudes y cualidades personales (Hidalgo et al. 2007; Lara y Rivas, 2009).

Las estrategias de aprendizaje como procesos mentales no son directamente observables, sin embargo, si pueden ser detectables. La elaboración y usos de cuestionarios en este sentido son de mucha utilidad, como en nuestro caso, para la autoevaluación de los propios procesos cognitivos y afectivos en relación con la comprensión lectora.

La evaluación-autoevaluación es condición esencial en el aprendizaje. La misma regularmente se asume como un elemento externo que influye desde la visión y posición del profesor como director del proceso, pero es un error soslayar sus potencialidades como condición interna. La evaluaciónautoevaluación es motor que activa de forma consciente el aprendizaje, ya que ella, como función de la dirección del proceso, constituye un elemento importante, pues está llamada a potenciar la autovaloración del estudiante durante el aprendizaje, sus fortalezas y debilidades, sus preferencias y expectativas, los procedimientos empleados, el autocontrol de lo aprendido y la regulación de la actividad. Ésta será cualitativamente superior en tanto esté concebida de tal forma que contribuya a la autonomía del alumnado y a que valore cómo aprender (Gil y Padilla, 2009; Milán et al, 2009).

La evaluación educativa o formativa, según González (2009), es aquella que contribuye al desarrollo del estudiante en correspondencia con los rasgos fundamentales del proceso de formación del futuro profesional y con los objetivos sociales que plantea esa formación en nuestra sociedad. El cuestionario que presentamos tiene este carácter por cuanto con el mismo se persigue que:

- Actúe como orientador y regulador del proceso de comprensión o competencia lectora.

- Diagnostique y valore los estados y progresos del alumnado con respecto al uso de estrategias de lectura a diferentes niveles. 
- Constituya un instrumento de evaluación individual del proceso de lectura no solo para el estudiante, sino también para el profesorado y sirva para la reflexión del progreso colectivo en esta variable en el grupo.

- Constituya un objeto de aprendizaje, por cuanto el estudiante lo utiliza como instrumento de autovaloración y autorregulación.

- Explore las vías que permitan valorar integralmente el proceso de comprensión del alumnado.

- Cumpla las condiciones (relativas a finalidades, tipo de exigencias, formas de realización) que favorezcan el desarrollo de valores esenciales como la honestidad, la responsabilidad y el colectivismo.

- Regule y oriente al estudiante hacia la profundización en el estudio mediante el desarrollo de su motivación, la conciencia de su importancia personal y social y la formación de estrategias de aprendizaje. (González, 2009, p.6).

Fitzpatrick (2006), Brodie e Irving (2007) y Gil y Padilla (2009) indican que la participación del alumnado en el proceso evaluativo es imprescindible, ya que desarrolla el pensamiento analítico, el espíritu crítico, la emisión de juicios y criterios y la autodeterminación. Así pues, la evaluación se convierte en un elemento fundamental e inseparable de la práctica educativa, y permite dar al estudiante y profesorado la información de cada momento y realizar los juicios de valor necesarios para la orientación y toma de decisiones respecto al proceso de enseñanza-aprendizaje. Estas habilidades están en congruencia con el propio desarrollo de las estrategias de lectura, ya que forman parte de los indicadores seleccionados en el cuestionario de evaluación.

El estudio de las variables objeto de investigación, nos posibilitó presentar un instrumento dirigido a evaluar las estrategias del alumnado durante el procesamiento de información que respondiera a las exigencias de los planes de estudios en el nivel universitario y que evaluara, principalmente, sus resultados en términos cualitativos y cuantitativos, aunque tanto los números como las palabras no pueden describir ni interpretar y mucho menos medir con precisión toda la subjetividad humana.

Al seleccionar el cuestionario como instrumento para evaluar el uso de estrategias de lectura, se hace necesario fundamentar teóricamente las dimensiones e indicadores asumidos en su elaboración, lo que vendrá a fortalecer, con elementos teóricos sustanciales, su validez y fiabilidad. 


\section{DESCRIPCIÓN DE LOS PROCEDIMIENTOS EN LA ELABORACIÓN DEL CUESTIONARIO DE EVALUACIÓN DE LAS ESTRATEGIAS DE LECTURA}

El cuestionario de evaluación-autoevaluación de estrategias de lectura constituye una nueva aportación en este campo. Para su elaboración se siguió el siguiente proceso:

1. Se delimitó como campo de estudio el proceso de lectura y dentro de su complejidad se seleccionaron diferentes acciones y operaciones o estrategias a niveles de traducción, interpretación y extrapolación que respondieran a los propósitos esenciales de nuestros programas y planes de estudio. También se seleccionaron operaciones dirigidas a establecer los metaconocimientos de los procesos mentales del individuo.

2. Se realizó una lista de indicadores y categorías que cubrieran la esencia de las estrategias de lectura.

3. Se seleccionaron para cada uno de los indicadores tres ítems o más que respondieran a lo que se proponía medir utilizando una escala Lickert con seis opciones, quedando de esta forma conformado el cuerpo esencial del instrumento. También se definió la forma de evaluación para facilitarle los resultados al propio alumnado.

4. Se aplicaron métodos empíricos y estadísticos para establecer las características técnicas de fiabilidad y validez de contenido y de constructo del cuestionario de evaluación de las estrategias de lectura.

\begin{tabular}{|l|l|c|}
\hline \multicolumn{1}{|c|}{ DIMENSIONES } & \multicolumn{1}{|c|}{ INDICADORES } & ITEMS \\
\hline $\begin{array}{l}\text { A. CONOCIMIENTO } \\
\text { PREVIO }\end{array}$ & $\begin{array}{l}\text { - Trazarse un propósito para la lectura. } \\
\text { - Activar los conocimientos previos. } \\
- \text { Tener conciencia de los propios procesos } \\
\text { de pensamiento. }\end{array}$ & $1-13$ \\
\hline $\begin{array}{l}\text { B. NIVEL DE } \\
\text { TRADUCCIÓN }\end{array}$ & $\begin{array}{l}\text { - Comprender globalmente. } \\
\text { - Reconocer significados: explícitos, implícitos } \\
\text { - Remplementarios. } \\
- \text { Reconocer la estructura textual. } \\
\text { - Resumizar, identificar y jerarquizar ideas. }\end{array}$ & $14-39$ \\
\hline $\begin{array}{l}\text { C. NIVEL DE } \\
\text { INTERPRETACIÓN }\end{array}$ & $\begin{array}{l}\text { - Inferir información implícita. } \\
- \text { Valorar la información. } \\
\text { - Emitir juicios y criterios. }\end{array}$ & $40-52$ \\
\hline
\end{tabular}




\begin{tabular}{|l|l|c|}
\hline \multicolumn{1}{|c|}{ DIMENSIONES } & \multicolumn{1}{|c|}{ INDICADORES } & ITEMS \\
\hline $\begin{array}{l}\text { D. NIVEL DE } \\
\text { EXTRAPOLACIÓN }\end{array}$ & $\begin{array}{l}\text { - Extender la información. } \\
\text { - Analizar situaciones similares. } \\
\text { - Resolver problemas. }\end{array}$ & $52-66$ \\
\hline
\end{tabular}

Tabla 1. Resumen de las dimensiones e indicadores seleccionados

Una vez establecidas las dimensiones y los indicadores correspondientes, se procedió a la definición de los diferentes ítems que iban a configurar el cuestionario que se puede observar a continuación:

\section{CUESTIONARIO DE EVALUACIÓN DE ESTRATEGIAS UTILIZADAS DURANTE LA COMPRENSIÓN DE LECTURA EN UNA LENGUA EXTRANJERA}

Durante el proceso de lectura en una lengua extranjera, el ser humano pone en funcionamiento una serie de estrategias de aprendizaje, tales como: estrategias metacognitivas o procedimentales, estrategias cognitivas o conceptuales de varios tipos, así como estrategias de interacción social, que les van a servir para una mejor compresión de lo que está leyendo. Con este cuestionario tratamos de que reflexiones sobre lo que te proponemos y nos contestes en qué medida tú lo haces.

En los apartados (A -B -C -D) que se incluyen a continuación, están recogidas las diversas estrategias que pueden ser utilizadas en el proceso de comprensión de una lectura. Léelas cuidadosamente y valóralas de 1 a 6 , según los siguientes parámetros:

1. No lo hago nunca.

2. Lo hago muy pocas veces.

3. Lo hago algunas veces.

4. Lo hago bastantes veces.

5. Lo hago muchas veces.

6. Lo hago siempre.

Elige la puntuación, marcando con una X, la que corresponde a lo que tú haces realmente y no a lo qué piensas que deberías hacer o a lo que hacen los demás.

Por favor no dejes ninguna respuesta en blanco. 


\section{A. CONOCIMIENTO PREVIO}

Cuando leo un texto en idioma inglés:

\begin{tabular}{|c|c|c|c|c|c|c|}
\hline Ítems & 1 & 2 & 3 & 4 & 5 & 6 \\
\hline 1. Tengo definido el propósito de mi lectura. & & & & & & \\
\hline 2. Sé qué voy a leer y para qué voy a leerlo. & & & & & & \\
\hline 3. Leo con un objetivo determinado. & & & & & & \\
\hline $\begin{array}{l}\text { 4. Pienso que tengo los conocimientos previos necesa- } \\
\text { rios para enfrentar un texto, (conocimientos tanto } \\
\text { lingüísticos, del tema, como generales del mundo). }\end{array}$ & & & & & & \\
\hline $\begin{array}{l}\text { 5. Creo que domino el vocabulario necesario relaciona- } \\
\text { do con el tema del texto. }\end{array}$ & & & & & & \\
\hline $\begin{array}{l}\text { 6. Creo que domino las estructuras lingüísticas elemen- } \\
\text { tales del idioma inglés para enfrentar la lectura. }\end{array}$ & & & & & & \\
\hline $\begin{array}{l}\text { 7. Creo tener suficientes conocimientos generales para } \\
\text { entender información complementaria. }\end{array}$ & & & & & & \\
\hline $\begin{array}{l}\text { 8. Utilizo conscientemente mis propios pasos o procedi- } \\
\text { mientos para procesar la información de un texto. }\end{array}$ & & & & & & \\
\hline $\begin{array}{l}\text { 9. Pienso en cómo procesar la información, trazo } \\
\text { acciones a seguir. }\end{array}$ & & & & & & \\
\hline 10. Proceso la información sin ayuda. & & & & & & \\
\hline $\begin{array}{l}\text { 11. Proceso la información siguiendo una secuencia } \\
\text { lógica. }\end{array}$ & & & & & & \\
\hline $\begin{array}{l}\text { 12. Proceso la información con destreza (independencia, } \\
\text { rapidez, calidad). }\end{array}$ & & & & & & \\
\hline $\begin{array}{l}\text { 13. Rectifico mis pasos o procedimientos cuando algo no } \\
\text { sale bien. }\end{array}$ & & & & & & \\
\hline
\end{tabular}

\section{B. NIVEL DE TRADUCCIÓN}

Cuando realizo una lectura exploratoria o global:

\begin{tabular}{|l|l|l|l|l|l|l|}
\hline \multicolumn{1}{|c|}{ Ítems } & 1 & 2 & 3 & 4 & 5 & 6 \\
\hline 14. Anticipo el contenido del texto por su título. & & & & & & \\
\hline $\begin{array}{l}\text { 15. Determino la información general contenida en el } \\
\text { texto al leer el primer párrafo y/o el último. }\end{array}$ & & & & & & \\
\hline $\begin{array}{l}\text { 16. Determino la información general contenida en el } \\
\text { texto al pasar la vista rápidamente por el texto. }\end{array}$ & & & & & & \\
\hline
\end{tabular}




\begin{tabular}{|c|c|c|c|c|c|}
\hline Ítems & 1 & 2 & 3 & 4 & 5 \\
\hline $\begin{array}{l}\text { 17. Pienso en el mensaje o significado que transmite el } \\
\text { título. }\end{array}$ & & & & & \\
\hline 18. Reconozco la información explícitamente expresada. & & & & & \\
\hline 19. Infiero la información que intenta transmitir el autor. & & & & & \\
\hline 20. Relaciono e integro ideas. & & & & & \\
\hline 21. Reconozco las características del texto. & & & & & \\
\hline $\begin{array}{l}\text { 22. Reconozco la función comunicativa del texto, su } \\
\text { estructura textual. }\end{array}$ & & & & & \\
\hline $\begin{array}{l}\text { 23. Reconozco los conectores o palabras relacionantes en } \\
\text { un texto. }\end{array}$ & & & & & \\
\hline 24. Reconozco la relación que expresan. & & & & & \\
\hline 25. Reconozco la estructura de los párrafos. & & & & & \\
\hline $\begin{array}{l}\text { 26. Reconozco la estructura textual del texto como con- } \\
\text { junto. }\end{array}$ & & & & & \\
\hline $\begin{array}{l}\text { 27. Encuentro información relacionada a una idea que } \\
\text { está esparcida dentro del texto. }\end{array}$ & & & & & \\
\hline $\begin{array}{l}\text { 28. Reconozco la posición de las ideas de un texto } \\
\text { tradicional. }\end{array}$ & & & & & \\
\hline $\begin{array}{l}\text { 29. Identifico ideas principales, ideas secundarias y } \\
\text { detalles. }\end{array}$ & & & & & \\
\hline $\begin{array}{l}\text { 30. Reconozco cuándo una idea está contenida dentro de } \\
\text { otra. }\end{array}$ & & & & & \\
\hline $\begin{array}{l}\text { 31. Reconozco cuándo una idea generaliza o incluye } \\
\text { otras ideas. }\end{array}$ & & & & & \\
\hline $\begin{array}{l}\text { 32. Identifico un grupo de ideas que están relacionadas } \\
\text { con otra más general. }\end{array}$ & & & & & \\
\hline 33. Identifico los detalles de una idea. & & & & & \\
\hline 34. Jerarquizo las ideas y las sintetizo. & & & & & \\
\hline 35. Organizo correctamente las ideas en el resumen. & & & & & \\
\hline 36. Suprimo la información que no es importante. & & & & & \\
\hline $\begin{array}{l}\text { 37. Integro ideas fundamentales comunes en una más } \\
\text { amplia. }\end{array}$ & & & & & \\
\hline 38. Selecciono solo lo esencial. & & & & & \\
\hline 39. Redacto la información sintetizada. & & & & & \\
\hline
\end{tabular}




\section{NIVEL DE INTERPRETACIÓN}

Cuando valoro críticamente la información leída:

\begin{tabular}{|c|c|c|c|c|c|c|}
\hline Ítems & 1 & 2 & 3 & 4 & 5 & 6 \\
\hline 40. Capto información implícita. & & & & & & \\
\hline 41. Infiero el tono, la atmósfera del texto. & & & & & & \\
\hline 42. Reconozco lo que el escritor intenta transmitir. & & & & & & \\
\hline $\begin{array}{l}\text { 43. Reconozco cuándo el emisor se basa en sus propios } \\
\text { juicios. }\end{array}$ & & & & & & \\
\hline 44. Reconozco cuándo el autor se basa en hechos. & & & & & & \\
\hline $\begin{array}{l}\text { 45. Valoro mis conocimientos sobre el tema durante la } \\
\text { lectura. }\end{array}$ & & & & & & \\
\hline 46. Extraigo conclusiones de mis inferencias. & & & & & & \\
\hline 47. Comparo esa información con otras fuentes. & & & & & & \\
\hline $\begin{array}{l}\text { 48. Determino cuándo el autor aporta nuevos elementos } \\
\text { sobre lo que sé del tema. }\end{array}$ & & & & & & \\
\hline $\begin{array}{l}\text { 49. Reconozco cuándo el autor ofrece una fundamenta- } \\
\text { ción convincente. }\end{array}$ & & & & & & \\
\hline 50. Establezco y emito mis propios puntos de vista. & & & & & & \\
\hline 51. Emito mis puntos de vistas y los argumento. & & & & & & \\
\hline
\end{tabular}

\section{NIVEL DE EXTRAPOLACIÓN}

Cuando aplico información leída:

\begin{tabular}{|c|c|c|c|c|c|c|}
\hline Ítems & 1 & 2 & 3 & 4 & 5 & 6 \\
\hline $\begin{array}{l}\text { 52. Integro información del texto a mis conocimientos } \\
\text { previos sobre el tema. }\end{array}$ & & & & & & \\
\hline $\begin{array}{l}\text { 53. Aplico la información leída a otros contextos de la } \\
\text { vida. }\end{array}$ & & & & & & \\
\hline $\begin{array}{l}\text { 54. Identifico los nexos y relaciones entre la información } \\
\text { contenida en el texto y lo que conozco del tema. }\end{array}$ & & & & & & \\
\hline $\begin{array}{l}\text { 55. Reconozco rasgos comunes de la situación dada con } \\
\text { situaciones similares de la realidad. }\end{array}$ & & & & & & \\
\hline $\begin{array}{l}\text { 56. Encuentro nexos y relaciones entre la información } \\
\text { leída y otros contextos. }\end{array}$ & & & & & & \\
\hline $\begin{array}{l}\text { 57. Determino el futuro de situaciones similares de la } \\
\text { realidad de no dársele solución inmediata. }\end{array}$ & & & & & & \\
\hline
\end{tabular}




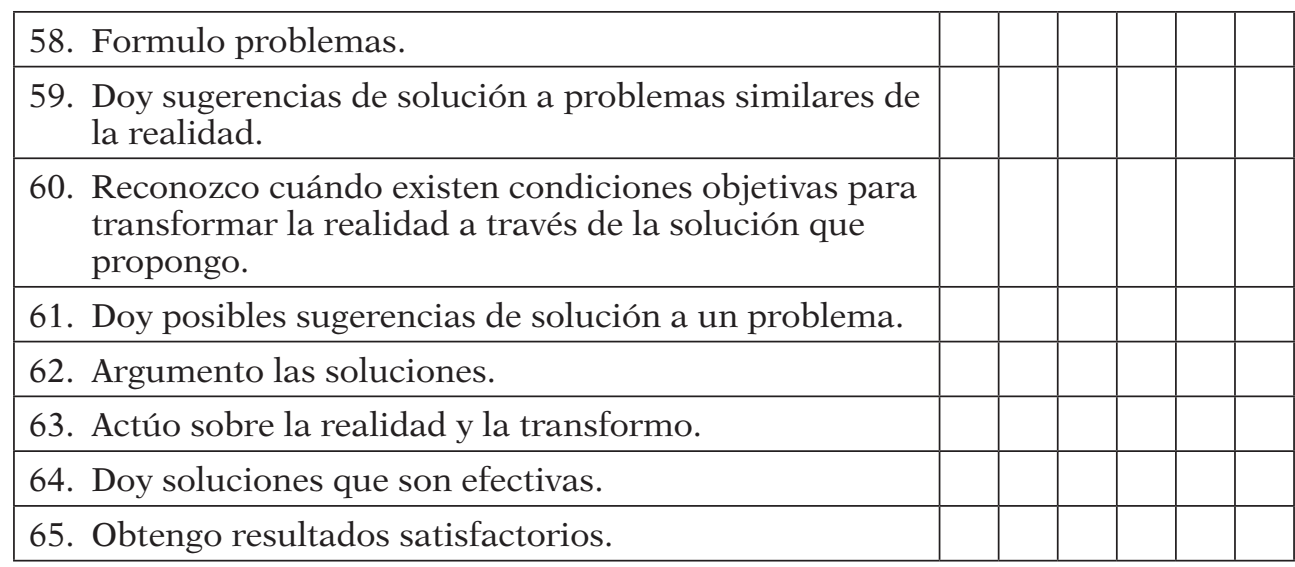

\section{PUNTUACIÓN}

\begin{tabular}{|c|l|}
\hline$\bullet$ & Pon tu puntuación en la casilla que hay al lado de cada número. \\
\hline$\bullet$ & $\begin{array}{l}\text { Suma la puntuación de cada apartado y divide el total obtenido por el } \\
\text { número de items: obtendrás así tu media personal para cada apartado. }\end{array}$ \\
\hline$\bullet$ & $\begin{array}{l}\text { Cada apartado corresponde a un grupo de estrategias. Lee el resumen } \\
\text { y compara tus medias personales con la clave para saber qué grupo de } \\
\text { estrategias sueles utilizar con más frecuencia. }\end{array}$ \\
\hline
\end{tabular}

\section{Clave de valoración de las estrategias utilizadas}

\begin{tabular}{|l|l|}
\hline Uso muy frecuente & 4,5 a 5,0 (lo hago siempre o casi siempre) \\
\hline Uso frecuente & 3,5 a 4,4 (lo hago a menudo) \\
\hline Uso moderado & 2,5 a 3,4 (lo hago a veces) \\
\hline Uso limitado & 1,5 a 2,4 (generalmente no lo hago) \\
\hline Uso muy limitado & 1,0 a 1,4 (no lo hago casi nunca / nunca) \\
\hline
\end{tabular}

Evaluar el uso de tus estrategias de lectura te ha permitido descubrir cuáles utilizas con mayor frecuencia para leer en una lengua extranjera. También te ha permitido darte cuenta de que durante el proceso de comprensión en una lengua extranjera, recurres a procedimientos que manejas cuando lees en la lengua materna y que la lectura en lengua extranjera va más allá de la simple decodificación de un texto. Conjuntamente, el conocimiento personal del uso de las estrategias de lectura, te facilita conocer tus potencialidades y debilidades durante el procesamiento de la información de un texto escrito y como consecuencia de ello, perfeccionar las limitaciones. 
Por último, ten presente también que una buena utilización de las estrategias de comprensión depende de varios factores: edad, personalidad, nivel de conocimientos alcanzados en la lengua extranjera, objetivos y necesidades de aprendizaje, experiencias anteriores, etc. Sin embargo, en la lista hay seguramente estrategias que tú no has utilizado hasta ahora y que te pueden ser de mucha utilidad.

\section{CARACTERÍSTICAS TÉCNICAS DEL CUESTIONARIO DE ESTRATEGIAS DE LECTURA: SU FIABILIDAD Y VALIDEZ}

La validez y la fiabilidad son constantes que deben estar siempre presentes en el proceso de elaboración de un cuestionario sobre todo, como en nuestro caso, cuando se miden operaciones que el individuo realiza generalmente de forma inconsciente, como las estrategias de aprendizaje. Por todo ello, se hace necesario ser cuidadoso y riguroso en el proceso de elaboración y de validación.

\section{Análisis y estudio de la validez de contenido}

En principio se distribuyó el cuestionario preparado para facilitar su análisis a los jueces que actuaron como expertos entre los que figuraban: Profesorado del Departamento de Métodos de Investigación y Diagnóstico en Educación, del Departamento de Lengua Extrajera y Literatura y del Departamento de Psicología Evolutiva y de la Educación, así como personas de otros estamentos tales como: Asesores de los Centros de Profesorado e Inspectores de los Servicios Técnicos de Inspección. Los jueces debían seleccionar de entre una escala de 4 opciones: $1=$ Nada; $2=$ Poco; $3=$ Bastante; $4=$ Totalmente. Valorando con ello el grado de relación entre los ítems y las dimensiones establecidas.

La valoración dada por los diez jueces expertos quedó reflejada entre los rangos: bastante y totalmente. Con respecto a su univocidad y pertinencia todos estuvieron de acuerdo en que los ítems eran claros, concisos, concretos, se entendían bien y eran pertinentes para el estudio.

Estos resultados finalmente, confirman que los expertos concuerdan en que las preguntas contenidas dentro del cuestionario diseñado, corresponden al objetivo esencial de nuestro instrumento y de nuestro estudio, lo que permite corroborar su validez de contenido.

\section{Análisis y estudio de la validez de constructo}

El análisis factorial es aconsejable para estudiar la estructura de los cuestionarios de manera que podamos afirmar que determinados ítems se 
explican mejor desde una dimensión que desde otra, incluso nos sugiere aquellos ítems que puedan ser eliminados por escasa consistencia interna en correspondencia con otros de las diferentes dimensiones seleccionadas (Hidalgo, 2003).

Por ello aplicamos análisis factorial, obteniendo los siguientes resultados:

- Matriz de correlaciones. Los determinantes de la matriz fueron bajos, lo que confirmó que las correlaciones no son debidas al azar, demostrando así la intercorrelación entre las dimensiones del instrumento.

- La prueba de esfericidad de Bartlett. Probó la no significatividad de la hipótesis nula de que la matriz de correlaciones era una matriz de identidad y que, por lo tanto, tiene sentido aplicar el análisis factorial.

- La medida de adecuación de muestreo KMO (Kaiser-Meyer-Olkin). En nuestro caso, la medida de adecuación de muestreo KMO estuvo entre los coeficientes 1 y 0,8 catalogados como "maravillosos", y "meritorios" respectivamente. Esto implica que las correlaciones entre pares de variables pueden explicarse por otras variables y que, por lo tanto, resulta adecuada la aplicación del análisis factorial.

- Determinación de las comunalidades: Los valores que aparecen en la columna de extracción se alejan de cero, lo que nos indicó que la variable quedaba totalmente explicada por su componente al acercarse a un valor de uno.

- Método de extracción: Para cada una de las cuatro dimensiones se aplicó: Varianza total, Gráfico de Sedimentación y la Matriz de Componentes, a través de los cuales se corroboró que los ítems de cada apartado se podían resumir, o sea, se podían agrupar en componentes que explicaran la mayoría de la varianza observada en las respuestas ofrecidas por los encuestados, lo que implica que todas las variables estarían representadas de forma coherente.

- Método de rotación: El procedimiento utilizado fue una rotación ortogonal Varimax para cada una de las dimensiones. Al tener estas un índice de correlación interfactores bajo, queda claro que los componentes están relacionados entre sí. 
Estos resultados nos permitieron realizar un estudio confirmatorio para cada dimensión y establecer que los ítems se ajustan a cada uno de los indicadores, lo que demuestra que existe consistencia interna entre los ítems del instrumento.

\section{Fiabilidad del instrumento}

Para obtener la fiabilidad de nuestro instrumento se aplicó el Alpha de Crombach, tanto para el cuestionario completo como para cada una de las dimensiones establecidas para conformar el mismo. Se administró el cuestionario a 119 estudiantes de segundo año, los cuales culminan la asignatura Inglés IV en el segundo semestre de este curso académico (Manzano, 2007).

Como se puede observar en la tabla, los resultados obtenidos muestran un coeficiente de Alpha de Crombach de altísima fiabilidad.

\begin{tabular}{|c|}
\hline $\mathrm{N}$ of Cases $=119,0 \quad \mathrm{~N}$ of Items $=65$ \\
\hline Alpha $=0,9688$ \\
\hline
\end{tabular}

Tabla 2. Fiabilidad por Alpha de Crombacl

\section{A MODO DE REFLEXIÓN}

La lectura es esencial durante el proceso de enseñanza-aprendizaje porque en ella intervienen procesos mentales de alto nivel, desarrolla destrezas y capacidades y posee potencialidades para ser explorada desde diferentes direcciones, sobre todo por parte de los educadores de las diferentes ramas del saber, pues debemos tener conciencia de que la lectura está presente cotidianamente en la docencia y que por lo tanto, es un problema que urge a todos perfeccionar desde nuestras propias perspectivas y posibilidades. De ahí que un trabajo consciente, interdisciplinar, sistemático, cohesionado y armónico con la comprensión, permitiría que el alumnado se apropie de algoritmos imprescindibles para el procesamiento de la información, como vía de solución para contribuir a la independencia cognoscitiva.

El dominio de destrezas y desarrollo de capacidades en la lectura, contribuye al logro de la autonomía del estudiante durante la adquisición de los conocimientos en una lengua extranjera. Para lograr esas destrezas y capacidades es necesario un trabajo consciente por parte del aprendiz y también, la reflexión colectiva en el avance personal de las estrategias a diferentes niveles de comprensión para que estas se desarrollen de manera integral. 
El instrumento que se presenta permite su utilización por apartados en correspondencia con los objetivos que persigamos en nuestra práctica pedagógica. Posibilita, además, que los sujetos del proceso de enseñanzaaprendizaje intercambien ideas, utilicen el proceso de lectura como medio y método de enseñanza y aprendizaje de forma eficaz y eficiente, lo que implica construir significados y aplicarlos sistemáticamente para adquirir rapidez e independencia en el manejo de la información.

«Los medios pueden favorecer la actividad sujeto-sujeto y la interacción sujeto-sujeto, cuando representan un eslabón de enlace con el acervo cultural con el que el proceso de enseñanza-aprendizaje ha de pertrechar a los estudiantes» (Zilberstein y Collazo, 2009, p. 338). El cuestionario de evaluación de lectura está en función de dicha actividad cognoscitiva y afectiva ,por lo que cumple varias funciones:

- Instructiva: Promueve la apropiación de los conocimientos, el desarrollo de habilidades y permite estudiar los objetos, fenómenos o procesos lo más objetivamente posible.

- Cibernética: Influye en las respuestas del estudiante, regula el proceso de enseñanza-aprendizaje, permite conocer las preferencias del estudiante, su motivación, manera de actuar.

- Formativa: Influye en su formación, convicciones y valores. Eleva su cultura, su cosmovisión y conocimiento de sí mismo.

- Recreativa: Favorece la distracción, permite el cambio de actividad, favorece la utilización de juegos.

- Desarrolladora: Favorece la integralidad de la personalidad del estudiante.

- De control: Propicia la observación, el autocontrol y la valoración del aprendizaje.

- Motivadora, innovadora y creadora: Motiva y entusiasma por la apropiación del contenido y despierta intereses e inclinaciones hacia la actividad creadora.

Desde el punto de vista práctico-metodológico, el cuestionario presentado propone estrategias que pueden ser objetivos que propicien tareas o actividades relacionadas con los textos que se imparten durante la enseñanza y el aprendizaje de la lengua. Estas actividades posibilitan la construcción de significados y sentidos, el desarrollo crítico y creativo del pensamiento a 
través del procesamiento de la información y alejan al proceso enseñanzaaprendizaje de actividades mecánicas y reproductivas, al permitir al estudiante que:

- Activen sus conocimientos previos sobre el tema.

- Tengan conciencia del objetivo de la lectura y la tarea que van a realizar.

- Trabajen los tres significados que aporta un texto: el literal o explícito, el intencional o implícito y el cultural o complementario.

- Desarrollen procesos de análisis - síntesis.

- Reflexionen sobre sus propias respuestas para que conozcan sus procesos mentales y puedan utilizar estrategias de autocorrección.

- Tengan en cuenta sus opiniones.

- Reconozcan la utilidad de lo leído.

- Reflexionen sobre situaciones problemáticas relacionadas con el tema.

- Se motiven por la lectura de otros textos escritos sobre el tema.

- Tomen conciencia de los niveles de ayuda que necesitan. 


\section{REFERENCIAS BIBLIOGRÁFICAS}

Brodie, P. e Irving, K. (2007). Assessment in work-based learning: investigating a pedagogical approach to enhance student learning.Assessment \& Evaluation in Higher education, 32 (1), 11-19.

Farías, P.L. (2008). El uso del cuestionario como instrumento de enseñanza de la comprensión de textos en la escuela media.Revista Iberoamericana de Educación, 45 (5). Recuperado de http:// www.rieoie.org/boletin45_5.htm

Fitzpatrick, D. (2006). An evaluative case study of the dilemmas experienced in designing a self-assessment strategy for Community Nursing students. Assessment \& Evaluation in Higher Education, 31 (1), 37-53.

Fernández, A. M. (2009). La Comunicación y su importancia en la educación. Estilos de comunicación. En AAVV. fundamentos didácticos de la educación superior cubana. Selección de lecturas. La Habana: Félix Varela.

Freire, P. (2010). Pedagogía de la autonomía y otros textos. La Habana: Caminos.

Gil, J. y Padilla, M. T. (2009). La participación del alumnado universitario en la evaluación del aprendizaje. Educación XX1,12, 43-65.

González, M. (2000). Evaluación del aprendizaje en la enseñanza universitaria. Revista Pedagogía Universitaria. 5 (2) 1-121. Recuperado de http:// www.uclm.es/profesorado/ricardo/ docencia_e_investigacion/4/evaluacion_halcones.doc
González, M. (2009). La Evaluación del Aprendizaje, en AAVV. Preparación pedagógica para profesores de la Nueva Universidad Cubana. La Habana: Félix Varela.

Hidalgo, E. et al. (2007). Formación en Competencias de Ciudadanía Europea. Granada: Método.

Hidalgo, V. (2003). La mejora de un centro multicultural: de la ciudad autónoma de Ceuta. Tesis doctoral. Granada: Universidad de Granada.

Lara, S. y Rivas, S. (2009). Aprendizaje autorregulado y fomento de competencias en dos asignaturas de master a través del empleo de plantillas de evaluación, método del caso, role-playing y video digital. Revista Educación XX1, 12, 67-96.

Manzano, M. (2007). Estilos de aprendizaje, Estrategias de lectura y su relación con el rendimiento académico del inglés como lengua extranjera. Tesis del programa de Doctorado "Aportaciones Educativas en Ciencias Sociales y Humanas". Universidad de Granada-Universidad de Ciego de Ávila.

Milán, R.; Fuentes, H. C. y De la Peña, R. (2009). La Evaluación como un proceso participativo, en AAVV. Fundamentos didácticos de la educación superior cubana. Selección de lecturas. La Habana: Félix Varela.

Zilberstein, J. y Collazo, R. (2009). Los medios de enseñanza-aprendizaje, en AAVV. Fundamentos didácticos de la educación superior cubana. Selección de lecturas. La Habana: Félix Varela. 


\section{PALABRAS CLAVE}

Comprensión de lectura, estrategias de lectura, evaluación.

\section{KEYWORDS}

Reading comprehension, reading strategies, evaluation.

\section{PERFIL ACADÉMICO Y PROFESIONAL DE LOS AUTORES}

Eugenio Hidalgo Diez, Profesor Titular de Métodos de Investigación y Diagnóstico en Educación, Facultad de Ciencias de la Educación de la Universidad de Granada, donde imparte docencia. Vicedecano de Grado, Garantía de la Calidad y Acción Tutorial. Líneas de investigación: Evaluación de los procesos de enseñanza-aprendizaje y de las competencias. Gestión y evaluación de la Calidad en centros educativos. Análisis y Estudio de Políticas Educativas y de Calidad en contextos rurales.

Mirtha Manzano Díaz, Profesora Titular del Departamento de Idiomas de la Facultad de Ciencias Sociales y Humanísticas de la Universidad de Ciego de Ávila, Cuba. Vicedecana de Investigación y Postgrado. Imparte docencia e investiga en áreas del conocimiento relacionadas con la didáctica de las lenguas extranjeras, comprensión de lectura, competencia comunicativa y estrategias de aprendizaje. Dirige un proyecto de servicios y asesoría de traducción e interpretación.

Dirección de los Autores: EVHFOJP Hidalgo Díez

Campus La Cartuja s/n

18071 - Granada (ESPAÑA).

E-mail: eugenio@ugr.es

MJSU a Manzano Díaz

Pasaje B N.': 254 Entre: 2da y 3ra Reparto:

Ortiz,

Ciego de Ávila

CP: 65200 - Cuba

E-mail: mansano@humanidades.unica.cu alebroc@ciego.cult.cu.

Fecha Recepción del Artículo: 28. Agosto. 2011

Fecha modificación Artículo: 11. Abril. 2012

Fecha Aceptación del Artículo: 06. Junio. 2012

Fecha de Revisión para publicación: 24. Junio. 2013 